\title{
Geometric phase for non-Hermitian Hamiltonians and its holonomy interpretation
}

Hossein Mehri-Dehnavi' and Ali Mostafazadeh'

Citation: Journal of Mathematical Physics 49, 082105 (2008); doi: 10.1063/1.2968344

View online: http://dx.doi.org/10.1063/1.2968344

View Table of Contents: http://aip.scitation.org/toc/jmp/49/8

Published by the American Institute of Physics

\section{Articles you may be interested in}

Results on Certain Non-Hermitian Hamiltonians

8, (2004); 10.1063/1.1705118

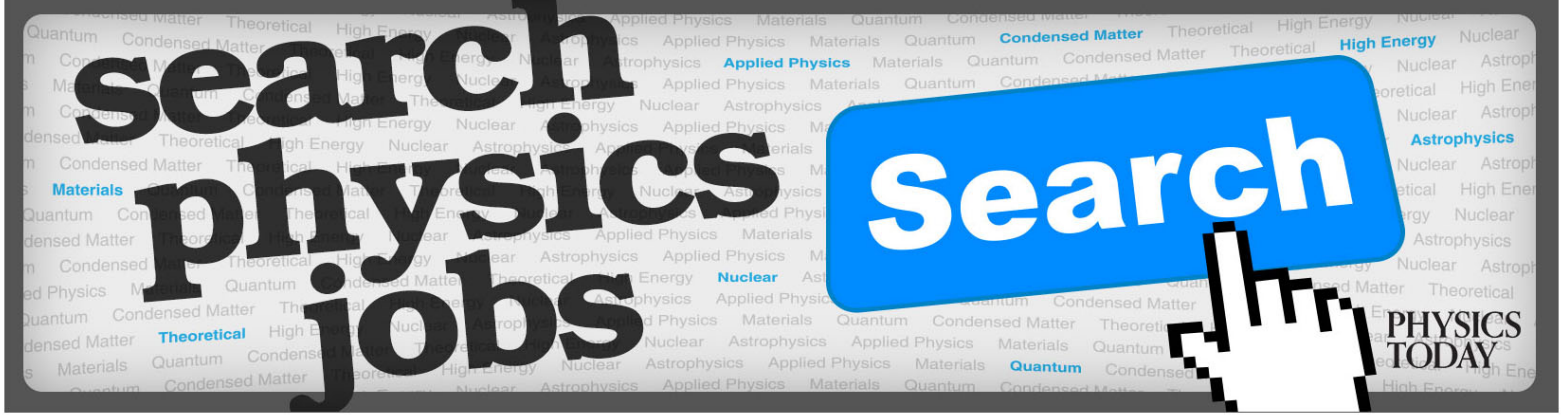




\title{
Geometric phase for non-Hermitian Hamiltonians and its holonomy interpretation
}

\author{
Hossein Mehri-Dehnavi ${ }^{1, a)}$ and Ali Mostafazadeh ${ }^{2, b)}$ \\ ${ }^{1}$ Department of Physics, Institute for Advanced Studies in Basic Sciences, \\ Zanjan 45195-1159, Iran \\ ${ }^{2}$ Department of Mathematics, Koç University, Rumelifeneri Yolu, Sariyer, \\ 34450 Istanbul, Turkey
}

(Received 27 June 2008; accepted 18 July 2008; published online 18 August 2008)

For an arbitrary possibly non-Hermitian matrix Hamiltonian $H$ that might involve exceptional points, we construct an appropriate parameter space $\mathfrak{M}$ and line bundle $L^{n}$ over $\mathfrak{M}$ such that the adiabatic geometric phases associated with the eigenstates of the initial Hamiltonian coincide with the holonomies of $L^{n}$. We examine the case of $2 \times 2$ matrix Hamiltonians in detail and show that, contrary to claims made in some recent publications, geometric phases arising from encircling exceptional points are generally geometrical and not topological in nature. (C) 2008 American Institute of Physics. [DOI: 10.1063/1.2968344]

\section{INTRODUCTION}

A state of a quantum system is said to be cyclic if its dynamical evolution yields the same state after a time $T$. For a pure state, this means that a corresponding state vector returns to the same ray in the Hilbert space but acquires a phase factor. The discovery that this phase factor consists of a dynamical and a geometrical part has important implications. ${ }^{1}$ The first general treatment of geometric phases that underlined their significance and made them a focus of intensive research in the past three decades is due to Berry, ${ }^{2}$ who considered Hermitian Hamiltonians undergoing adiabatic changes. Berry's adiabatic geometric phase admits a variety of generalizations. Among these are non-Abelian, ${ }^{3}$ nonadiabatic, ${ }^{4}$ noncyclic, ${ }^{5,6}$ classical, $^{7}$ and relativistic ${ }^{8}$ geometric phases. There have also been attempts to extend geometric phases to systems described by non-Hermitian Hamiltonians (Ref. 9). ${ }^{1}$

Geometric phases for systems having a Hermitian Hamiltonian have an interesting mathematical interpretation in terms of the holonomies of certain fiber bundles. ${ }^{1,4,10}$ The main purpose of the present article is to offer a comprehensive holonomy interpretation for the adiabatic geometric phases associated with non-Hermitian matrix Hamiltonians, particularly those involving exceptional points. It is known that in the presence of such points, an adiabatic periodic change of the Hamiltonian does not necessarily imply a cyclic evolution of its eigenstates. Even for the simple $2 \times 2$ Hamiltonians that have widely been investigated in recent years, ${ }^{11,12}$ one often needs to evolve the initial state vector for two complete periods of the Hamiltonian to achieve a cyclic evolution. This behavior makes a direct generalization of the results of Ref. 10 for general nonHermitian Hamiltonians intractable. In this article we offer a complete solution for the problem of the construction of the relevant parameter spaces and the fiber bundles that allow for a holonomy interpretation of the adiabatic geometric phases for general non-Hermitian matrix Hamiltonians. We also address the controversy related to the topological nature of these phases.

\footnotetext{
${ }^{1}$ An extensive list of references on geometric phases and their generalizations that appeared before the year 2000 is given in Ref. 45.

${ }^{a)}$ Electronic mail: mehrideh@iasbs.ac.ir.

b) Author to whom correspondence should be addressed. Electronic mail: amostafazadeh@ku.edu.tr.
} 
A significant difference between Hermitian and non-Hermitian Hamiltonians is the nature of their degeneracies. Hermitian Hamiltonians are diagonalizable, i.e., they admit a complete set of eigenvectors. This is true even at a degeneracy point where two or more eigenvalues coalesce. In contrast, for a non-Hermitian Hamiltonian, in addition to the usual degenerate points where the Hamiltonian is diagonalizable, there may exist points of degeneracy where one can no longer form a complete set of eigenvectors. We will respectively refer to these two types of degeneracy points as diabolic degeneracy points and exceptional degeneracy points. Following the terminology employed in Ref. 13, we will abbreviate the latter as exceptional points (EPs). ${ }^{2}$ Non-Hermitian Hamiltonians with EPs appear in the study of resonances, ${ }^{14,15}$ quantum chaos, ${ }^{13,16}$ quantum cosmology, ${ }^{17,18}$ magnetohydrodynamics, ${ }^{19}$ and various classical and quantum systems. ${ }^{12,20-26}$ Geometric phases associated with EPs have been the subject of various theoretical ${ }^{12,22,27,28}$ and experimental ${ }^{11,29}$ studies.

A controversial claim about the geometric phases associated with EPs is that they have a topological character. ${ }^{12}$ This means that these phases are invariant under smooth perturbations of the path traversed by the parameters of the system. The main characteristic feature of topological phases is that the phase angle is an integer multiple of $\pi$, i.e., the phase factor is merely a sign. This is the case for the adiabatic geometric phases for a real symmetric Hamiltonian. ${ }^{1,2,30,31} \mathrm{We}$ will argue that the claim pertaining the topological nature of EP-related geometric phases ${ }^{12}$ rests on the specific choice of the path of parameters made in the previous studies of the subject, and that it does not generally hold.

Some authors restrict their study of EP-related geometric phases to systems having a complex symmetric Hamiltonian. ${ }^{22,28}$ Often they claim that this restriction does not diminish the generality of their results because every square matrix is related to a symmetric matrix by a similarity transformation. One must however note that the similarity transformations that map an arbitrary time-dependent Hamiltonian into a symmetric one are generically time dependent. These timedependent similarity transformations induce an additional term in the Schrödinger equation that in general changes the value of the geometric phases and other physical quantities. Therefore, contrary to the above-mentioned claim, a complete treatment of EP-related geometric phases calls for the consideration of general possibly nonsymmetric Hamiltonians. ${ }^{3}$

In Ref. 32 the author discusses a straightforward extension of the approach of Ref. 10 to offer a holonomy interpretation of the adiabatic geometric phases for the non-Hermitian matrix Hamiltonians considered in Ref. 9. The treatment of Refs. 9 and 32 is based on the assumption that the eigenvalues of the Hamiltonian are single-valued functions of its parameters. This holds typically whenever the path of parameters of the Hamiltonian does not enclose an EP. The fact that this is not generally the case is at the root of the difficulties associated with devising a consistent holonomy interpretation of the adiabatic geometric phases for a general non-Hermitian Hamiltonian.

The organization of the article is as follows. In Sec. II, we outline the construction of the parameter spaces for quantum systems defined by non-Hermitian matrix Hamiltonians. In Sec. III, we construct the desired line bundle over the relevant parameter space. In Sec. IV, we calculate the adiabatic geometric phases for a general $2 \times 2$ complex matrix Hamiltonian and examine various specific examples. Here we also discuss the topological versus geometric nature of the EP-related geometric phases. Finally, in Sec. V we summarize our results and present our concluding remarks. The article includes two appendixes that are respectively devoted to an algebraic topologi-

\footnotetext{
${ }^{2}$ Kato, ${ }^{33}$ from whom the term "exceptional point" seems to have been adopted, identified an exceptional point with a one at which the number of distinct eigenvalues of the operator in question changes. For example, according to Kato, $x=1$ is an exceptional point of the $2 \times 2$ Hamiltonian $H[x]=\operatorname{diag}(x, 1)$. In our classification this point is a diabolic degeneracy point, where none of the interesting phenomena reported in the related literature ${ }^{11,12,20-22}$ occurs. In Ref. 23 EPs are called non-Hermitian degeneracies.

${ }^{3}$ The same is true about Hermitian Hamiltonians. Although every Hermitian Hamiltonian can be mapped via a similarity transformation to a real symmetric (in fact real diagonal) matrix, to investigate geometric phases for Hermitian Hamiltonians, one cannot confine one's attention to real symmetric or diagonal Hamiltonians.
} 
cal treatment of the parameter spaces constructed in the text and a derivation of an explicit expression for the adiabatic geometric phase for the cases that the curve in the parameter space does not lie in a single open patch but finitely many patches.

\section{CONSTRUCTION OF THE PARAMETER SPACE}

Consider an arbitrary $N \times N$ matrix Hamiltonian $H$. We can parametrize $H$ in terms of its $N^{2}$ complex entries. These correspond to $2 N^{2}$ real numbers $\left(X_{1}, X_{2}, \ldots, X_{2 N^{2}}\right)=: X$ that we can identify with the elements of $\mathbb{R}^{2 N^{2}}$. We will use the symbol $H[X]$ for the Hamiltonian to mark its $X$-dependence. The condition that $H[X]$ has $N$-distinct eigenvalues restricts $X$ to a connected open subset $M_{\max }$ of $\mathbb{R}^{2 N^{2}}$ that inherits the structure of a smooth $2 N^{2}$-dimensional real manifold from $\mathrm{R}^{2 N^{2}}$.

Let $M$ be a $d$-dimensional connected submanifold of $M_{\max }$ whose points $R$ are locally expressed by the coordinates $\left(R_{1}, R_{2}, \ldots, R_{d}\right)$. We shall consider the cases that the Hamiltonian is parametrized by the elements of $M$. We denote this using $H[R]$ in place of $H[X]$. Clearly, the largest possible choice for $M$ is $M_{\max }$ in which case $d=2 N^{2}$. If we consider a general Hermitian $N \times N$ matrix Hamiltonian, $d=N^{2}$ and $M$ is a submanifold of $\mathrm{R}^{N^{2}}$. For $N=2$, we have $M=\mathbb{R}$ $\times\left(\mathbb{R}^{3}-\{0\}\right)=\mathbb{R} \times \mathbb{R}^{+} \times S^{2}$, where $S^{2}:=\left\{(x, y, z) \in \mathbb{R}^{3} \mid x^{2}+y^{2}+z^{2}=1\right\}$.

Let $\mathfrak{I}$ be a (nonempty) indexing set, $\left\{U_{\alpha}\right\}_{\alpha \in \mathfrak{I}}$ be an open cover of $M$ such that $U_{\alpha}$ is diffeomorphic to $\mathbb{R}^{d}$, and there are single-valued smooth functions

$$
E_{1}^{\alpha}: U_{\alpha} \rightarrow \mathrm{C}, \quad E_{2}^{\alpha}: U_{\alpha} \rightarrow \mathrm{C}, \ldots, \quad E_{N}^{\alpha}: U_{\alpha} \rightarrow \mathrm{C},
$$

such that for all $R \in U_{\alpha}$ and all $j \in\{1,2, \ldots, N\}=: I_{N}, E_{j}^{\alpha}[R]$ is an eigenvalue of $H[R]$. Note that such an open cover of $M$ always exists ${ }^{33}$ and because $H[R]$ has a nondegenerate spectrum, it has $N$ distinct eigenvalues; for all $m, n \in I_{N}$,

$$
E_{m}^{\alpha}[R] \neq E_{n}^{\alpha}[R] \text { if and only if } m \neq n .
$$

This implies the existence of a complete biorthonormal system $\left\{\left|\psi_{j}^{\alpha}[R]\right\rangle,\left|\phi_{j}^{\alpha}[R]\right\rangle\right\}$ of the Hilbert space $\mathcal{H}$ that satisfies

$$
H[R]\left|\psi_{j}^{\alpha}[R]\right\rangle=E_{j}^{\alpha}[R]\left|\psi_{j}^{\alpha}[R]\right\rangle, \quad H^{\dagger}[R]\left|\phi_{j}^{\alpha}[R]\right\rangle=E_{j}^{* \alpha}[R]\left|\phi_{j}^{\alpha}[R]\right\rangle, \quad\left\langle\phi_{j}^{\alpha}[R] \mid \psi_{k}^{\alpha}[R]\right\rangle=\delta_{j, k} .
$$

Here $\alpha \in \mathfrak{I}$ and $j, k \in I_{N}$ are arbitrary, $\dagger$ and $*$, respectively, denote the adjoint and complex conjugates, and $\delta_{m, n}$ stands for the Kronecker delta symbol. It is important to note that $\left|\psi_{j}^{\alpha}[R]\right\rangle$ and $\left|\phi_{j}^{\alpha}[R]\right\rangle$ define single-valued (smooth) functions mapping $U_{\alpha}$ to $\mathcal{H}$, namely, $\left|\psi_{j}^{\alpha}[\cdot]\right\rangle: U_{\alpha} \rightarrow \mathcal{H}$ and $\left|\phi_{j}^{\alpha}[\cdot]\right\rangle: U_{\alpha} \rightarrow \mathcal{H}$.

Conditions (3) do not determine the biorthonormal system $\left\{\left|\psi_{j}^{\alpha}[R]\right\rangle,\left|\phi_{j}^{\alpha}[R]\right\rangle\right\}$ uniquely, but they imply that any other biorthonormal system, $\left\{\left|\psi_{j}^{\prime \alpha}[R]\right\rangle,\left|\phi_{j}^{\prime \alpha}[R]\right\rangle\right\}$, fulfilling these conditions have the form

$$
\left|\psi_{j}^{\prime \alpha}[R]\right\rangle:=k_{j}^{\alpha}[R]\left|\psi_{j}^{\alpha}[R]\right\rangle, \quad\left|\phi_{j}^{\prime \alpha}[R]\right\rangle:=\frac{1}{k_{j}^{\alpha}[R]^{*}}\left|\phi_{j}^{\alpha}[R]\right\rangle .
$$

Here $j \in I_{N}$ is arbitrary and $k_{j}^{\alpha}: U_{\alpha} \rightarrow \mathrm{C}-\{0\}$ are smooth (single-valued) functions.

By definition, the (pure) states of a quantum system are rays (one-dimensional subspaces) of the Hilbert space $\mathcal{H}$. Every state is uniquely determined by any of its nonzero elements (state vectors). We use $\lambda_{\psi}$ to denote the state determined by a state vector $|\psi\rangle \in \mathcal{H}-\{0\}$, i.e.,

$$
\lambda_{\psi}:=\{c|\psi\rangle \mid c \in \mathbb{C}\} .
$$

States $\lambda_{\psi}$ are in one-to-one correspondence with the projection operators 


$$
\Lambda_{\psi}:=\frac{|\psi\rangle\langle\psi|}{\langle\psi \mid \psi\rangle} .
$$

In particular, we can characterize the eigenstates $\lambda_{j}^{\alpha}[R]$ of $H[R]$ that contain the eigenvectors $\left|\psi_{j}^{\alpha}[R]\right\rangle$ with the projection operators

$$
\Lambda_{j}^{\alpha}[R]:=\Lambda_{\psi_{j}^{\alpha}[R]}=\frac{\left|\psi_{j}^{\alpha}[R]\right\rangle\left\langle\psi_{j}^{\alpha}[R]\right|}{\left\langle\psi_{j}^{\alpha}[R] \mid \psi_{j}^{\alpha}[R]\right\rangle} .
$$

Note that as expected the projection operators $\Lambda_{j}^{\alpha}[R]$ are invariant under the "gauge transformations:" $\left|\psi_{j}^{\alpha}[R]\right\rangle \rightarrow\left|\psi_{j}^{\prime \alpha}[R]\right\rangle,\left|\phi_{j}^{\alpha}[R]\right\rangle \rightarrow\left|\phi_{j}^{\prime \alpha}[R]\right\rangle .^{1}$ This shows that the eigenstates $\lambda_{j}^{\alpha}[R]$ define single-valued functions $\Lambda_{j}^{\alpha}[\cdot]: U_{\alpha} \rightarrow \mathcal{L}(\mathcal{H})$, where $\mathcal{L}(\mathcal{H})$ is the set of linear operators acting in $\mathcal{H}$.

Similarly to the case of Hermitian Hamiltonians, ${ }^{2}$ every (parametrized) curve $C:[0, T] \rightarrow M$ defines a time-dependent Hamiltonian according to $H(t):=H[R(t)]$, where $R(t)$ stands for the coordinates of $C(t)$ in $U_{\alpha}$ and we have identified the parameter $t \in[0, T]$ of the curve $C$ with time. ${ }^{4}$ We will consider the dynamics generated by the Hamiltonian $H(t)$ via the Schrödinger equation

$$
H(t)|\Psi(t)\rangle=i \frac{d}{d t}|\Psi(t)\rangle
$$

where $|\Psi(t)\rangle$ is an evolving state vector.

We say that $H(t)$ generates an adiabatic time evolution if the eigenstates $\lambda_{j}^{\alpha}[R(0)]$ of $H(0)$ evolve into the eigenstates $\lambda_{j}^{\alpha}[R(t)]$ of $H(t)$ (with the same spectral label $j$ and for all $t \in[0, T]$ and $\alpha \in \mathfrak{I})$, i.e., given $\lambda_{\Psi(0)}=\lambda_{j}^{\alpha}[R(0)]$ we have $\lambda_{\Psi(t)}=\lambda_{j}^{\alpha}[R(t)] .{ }^{14,32,34}$ It is not difficult to show that this requirement puts too severe a restriction on the Hamiltonian $H(t) .{ }^{5}$ Therefore, in practice, one requires that this condition be satisfied approximately.

If $C$ is a simply closed curve, $H(t)$ is a periodic Hamiltonian with period $T$. In addition, if $H(t)$ generates an adiabatic time evolution and $C$ lies entirely in $U_{\alpha}$ for some $\alpha \in \mathfrak{I}$, then the eigenstates $\lambda_{j}^{\alpha}[R(0)]$ of $H(0)$ are cyclic states and we may explore the corresponding adiabatic geometric phases. However, if $C$ cannot be contained in a single open patch of $M$, it may happen that for some $j \in I_{N}, \alpha, \beta \in \mathfrak{I}$, and $C\left(t_{\star}\right) \in U_{\alpha} \cap U_{\beta}, \lambda_{j}^{\alpha}\left[R\left(t_{\star}\right)\right] \neq \lambda_{j}^{\beta}\left[R\left(t_{\star}\right)\right]$. Because of the nondegeneracy of the spectrum of the Hamiltonian, this is equivalent to $E_{j}^{\alpha}\left[R\left(t_{\star}\right)\right] \neq E_{j}^{\beta}\left[R\left(t_{\star}\right)\right]$. However, since $E_{j}^{\alpha}\left[R\left(t_{\star}\right)\right]$ is an eigenvalue of $H\left[R\left(t_{\star}\right)\right]$ regardless of whether we identify $R\left(t_{\star}\right)$ with coordinates of $C\left(t_{\star}\right)$ associated with the patch $U_{\alpha}$ or $U_{\beta}$, there should be some $k \in I_{N}-\{j\}$ such that $E_{j}^{\alpha}\left[R\left(t_{\star}\right)\right]$ $=E_{k}^{\beta}\left[R\left(t_{\star}\right)\right]$. In other words, it may happen that in certain points along the curve the eigenvalues (and consequently the eigenstates) with different spectral labels get swapped. If this happens $\lambda_{j}^{\alpha}[R(0)]$ may no longer be a cyclic state. The possibility of such a noncyclic adiabatic evolution has been ignored in the earlier investigations of the problem. ${ }^{9,14,32,34}$ It was brought to light when curves encircling EPs were considered.

The above-mentioned complication arises simply because the eigenvalues of the parameterdependent Hamiltonian are not single-valued functions on the parameter space $M$. This calls for the construction of appropriate parameter spaces for the problem. This is actually necessary for devising a holonomy interpretation for the adiabatic geometric phase of general non-Hermitian matrix Hamiltonians. We shall construct the desired parameter spaces as certain fiber bundles over $M$.

For each $\gamma \in \mathfrak{I}$, we define $s^{\gamma}: U_{\alpha} \rightarrow \mathrm{C}^{N}$ according to

${ }^{4}$ For brevity we shall use the same label for curve $C$ and its range $\{C(t) \mid t \in[0, T]\}$.

${ }^{5}$ For Hermitian Hamiltonians it implies that the eigenstates $\lambda_{j}^{\alpha}[R(0)]$ must actually be stationary. 


$$
s^{\gamma}(R):=\left(\begin{array}{c}
E_{1}^{\gamma}[R] \\
E_{2}^{\gamma}[R] \\
\vdots \\
E_{N}^{\gamma}[R]
\end{array}\right),
$$

and let $\alpha, \beta \in \mathfrak{I}$ be such that $U_{\alpha} \cap U_{\beta} \neq \varnothing$. For all $R \in U_{\alpha} \cap U_{\beta}$ and $k \in I_{N}, E_{k}^{\alpha}[R]$ is an eigenvalue of $H[R]$. This implies that there is a unique $j \in I_{N}$ such that $E_{k}^{\alpha}[R]=E_{j}^{\beta}[R]$. We can use this observation to infer the existence of a permutation $\sigma_{R}^{\beta, \alpha} \in \mathfrak{S}_{N}$ satisfying $j=\sigma_{R}^{\beta, \alpha}(k)$, where $\mathfrak{S}_{N}$ is the permutation group of $I_{N}$.

$\sigma_{R}^{\beta, \alpha}$ relates $s^{\alpha}$ and $s^{\beta}$ according to

$$
s^{\alpha}(R) \rightarrow s^{\beta}(R)=g\left(\sigma_{R}^{\beta, \alpha}\right) s^{\alpha}(R),
$$

where for every $\sigma \in \mathfrak{S}_{N}, g(\sigma) \in G L(N, \mathrm{C})$ is the $N \times N$ matrix defined by

$$
g(\sigma)\left(\begin{array}{c}
z_{1} \\
z_{2} \\
\vdots \\
z_{N}
\end{array}\right)=\left(\begin{array}{c}
z_{\sigma(1)} \\
z_{\sigma(2)} \\
\vdots \\
z_{\sigma(N)}
\end{array}\right), \text { for all }\left(\begin{array}{c}
z_{1} \\
z_{2} \\
\vdots \\
z_{N}
\end{array}\right) \in \mathbb{C}^{N},
$$

i.e., $g: \mathfrak{S}_{n} \rightarrow G L(n, C)$ gives the standard matrix representation of $\mathfrak{S}_{N}$.

Next, we define a complex vector bundle over $M$. We take $U_{\alpha} \times \mathrm{C}^{N}$ as the local charts and patch them together according to the following prescription.

(1) For each $(\alpha, \beta) \in \mathfrak{I}^{2}$ satisfying $U_{\alpha} \cap U_{\beta} \neq \varnothing$, we define $g_{\alpha \beta}: U_{\alpha} \cap U_{\beta} \rightarrow G L(N$, C) by

$$
\forall R \in U_{\alpha} \cap U_{\beta}, \quad g_{\alpha \beta}(R):=g\left(\sigma_{R}^{\alpha, \beta}\right) .
$$

(2) For all $R \in U_{\alpha} \cap U_{\beta}$ and $\vec{z} \in \mathrm{C}^{N}$, we identify the point $(R, \vec{z})$ in $U_{\beta} \times \mathrm{C}^{N}$ with the point $\left(R, g_{\alpha \beta}(R) \vec{z}\right)$ in $U_{\alpha} \times \mathbb{C}^{N}$.

By construction, this yields a fiber bundle $V$ with base manifold $M$, typical fiber $\mathrm{C}^{N}$, and structure group $G L(N, \mathrm{C}){ }^{35}$ For every $p \in V$, there are $U_{\alpha}, R \in U_{\alpha}$, and $\vec{z}^{\alpha} \in \mathrm{C}^{N}$ such that $p$ is represented by $\left(R, \vec{z}^{\alpha}\right)$. The projection map $\Pi: V \rightarrow M$ acts as $\Pi(p):=R$, and for each $\alpha \in \mathfrak{I}$ the function $\Phi_{\alpha}: \Pi^{-1}\left(U_{\alpha}\right) \rightarrow U_{\alpha} \times \mathbb{C}^{N}$ defined by

$$
\forall p \in \Pi^{-1}\left(U_{\alpha}\right), \quad \Phi_{\alpha}(p):=\left(R, \vec{z}^{\alpha}\right),
$$

provides a local trivialization of $V$, namely, $\left(U_{\alpha}, \Phi_{\alpha}\right)$. The functions $g_{\alpha \beta}$ introduced in (11) are the transition functions associated with these local trivializations.

Next, for every $\alpha, \beta \in \mathfrak{I}$ fulfilling $U_{\alpha} \cap U_{\beta} \neq \varnothing$, all $R \in U_{\alpha} \cap U_{\beta}$, and all $k \in I_{N}$, we identify the point $(R, k)$ in $U_{\beta} \times I_{N}$ with the point $\left(R, \sigma_{R}^{\alpha, \beta}(k)\right)$ in $U_{\alpha} \times I_{N}$. This defines a fiber bundle that we denote by $\mathcal{M}$. Let $\pi: \mathcal{M} \rightarrow M$ be the projection map for $\mathcal{M}$ and $\mathcal{R} \in \mathcal{M}$ be represented by $(R, k) \in U_{\alpha} \times I_{N}$. Then $\pi(\mathcal{R})=R$, the functions $\phi_{\alpha}: \pi^{-1}\left(U_{\alpha}\right) \rightarrow U_{\alpha} \times I_{N}$ defined by

$$
\forall p \in \pi^{-1}\left(U_{\alpha}\right), \quad \phi_{\alpha}(\mathcal{R})=(R, k),
$$

give a set of local trivializations $\left(U_{\alpha}, \phi_{\alpha}\right)$ of $\mathcal{M}$, and $\mathfrak{g}_{\alpha \beta}: U_{\alpha} \cap U_{\beta} \rightarrow \mathfrak{S}_{N}$ defined by

$$
\forall R \in U_{\alpha} \cap U_{\beta}, \quad \mathfrak{g}_{\alpha \beta}(R):=\sigma_{R}^{\alpha, \beta},
$$

are the corresponding transition functions.

As a simple example consider the $2 \times 2$ Hamiltonians of the form 


$$
H=\left(\begin{array}{ll}
0 & 1 \\
z & 0
\end{array}\right), \quad z \in \mathrm{C}-\{0\} .
$$

We can conveniently parametrize $H$ by the polar coordinates $r:=|z| \in \mathbb{R}^{+}$and $\theta:=\arg (z) \in \mathbb{R}$. The role of the manifold $M$ is now played by the punctured plane $\mathbb{R}^{2}-\{0\}$. The eigenvalues of $H$ are double-valued functions on $M=\mathbb{R}^{2}-\{0\}$,

$$
E_{1}=-\sqrt{z}=-\sqrt{r} e^{i \theta / 2}, \quad E_{2}=\sqrt{z}=\sqrt{r} e^{i \theta / 2} .
$$

We can cover $M$ by an open covering consisting of the following three open subsets:

$$
\begin{gathered}
U_{1}:=\left\{r e^{i \theta \mid} r \in \mathbb{R}^{+}, \theta \in(-\pi / 2, \pi / 2)\right\}, \\
U_{2}:=\left\{r e^{i \theta \mid} \mid r \in \mathbb{R}^{+}, \theta \in(\pi / 6,7 \pi / 6)\right\}, \\
U_{3}:=\left\{r e^{i \theta \mid} \mid r \in \mathbb{R}^{+}, \theta \in(5 \pi / 6,11 \pi / 6)\right\} .
\end{gathered}
$$

The parameter space $\mathcal{M}$ is the double covering space ${ }^{36}$ of $M$ that is constructed as a fiber bundle with typical fiber $I_{2}=\{1,2\}$ and the following transition functions associated with the open cover $\left\{U_{1}, U_{2}, U_{3}\right\}$ :

$$
\begin{gathered}
\forall R \in U_{1} \cap U_{2}=\left\{r e^{i \theta} \mid r \in \mathbb{R}^{+}, \theta \in(\pi / 6, \pi / 2)\right\}, \quad \mathfrak{g}_{12}(R):=\mathrm{Id}, \\
\forall R \in U_{2} \cap U_{3}=\left\{r e^{i \theta} \mid r \in \mathbb{R}^{+}, \theta \in(5 \pi / 6,7 \pi / 6)\right\}, \quad \mathfrak{g}_{23}(R):=\mathrm{Id}, \\
\forall R \in U_{3} \cap U_{1}=\left\{r e^{i \theta \mid} \mid r \in \mathbb{R}^{+}, \theta \in(-\pi / 2,-\pi / 6)\right\}, \quad \mathfrak{g}_{31}(R):=\tau,
\end{gathered}
$$

where Id is the identity permutation, $\operatorname{Id}(k)=k$ for all $k \in I_{2}$, and $\tau$ is the transposition that swaps 1 and 2. It is not difficult to identify $\mathcal{M}$ with the double-sheeted Riemann surface over which the square root $\sqrt{z}$ is a single-valued function of $z$.

In general the situation is more complicated than the simple example we just considered. As the following example shows, in general there may exist closed curves in $\mathcal{M}$ along which some of the eigenvalues of the Hamiltonian are multiple-valued. Let

$$
H[z]:=\left(\begin{array}{cc}
z & 0 \\
0 & H_{1}[z]
\end{array}\right), \quad H_{1}[z]:=\left(\begin{array}{ll}
0 & 1 \\
z & 0
\end{array}\right), \quad z \in M:=\mathrm{C}-\{0, \pm 1\} .
$$

The parameter space $\mathcal{M}$ for the Hamiltonian $H[z]$ is a threefold covering space of $M$. It consists of two connected components that we denote by $\mathcal{M}_{1}$ and $\mathcal{M}_{2,3}$. $\mathcal{M}_{1}$ is a diffeomorphic copy of $M$, and $\mathcal{M}_{2,3}:=\mathcal{M}^{\prime}-\pi^{-1}\{ \pm 1\}$, where $\mathcal{M}^{\prime}$ is a copy of the parameter space of the Hamiltonian (13). Clearly the eigenvalue $E_{1}[z]:=z$ is a single-valued function on $\mathcal{M}_{1}$ and $\mathcal{M}$, and the eigenvalues $E_{2,3}[z]:= \pm \sqrt{z}$ are single-valued on $\mathcal{M}_{2,3}$. However, the latter are not single-valued on $\mathcal{M}$. To see this let $C_{1}:[0,2 \pi] \rightarrow M$ be the curve defined by $z(t)=2 e^{i t}$, for all $t \in[0,2 \pi]$, and $\mathcal{C}_{1}:[0,2 \pi]$ $\rightarrow \mathcal{M}$ be the curve contained in $\mathcal{M}_{1}$ that projects onto $C_{1}$ under $\pi . \mathcal{C}_{1}$ is a closed curve in $\mathcal{M}$. Clearly $E_{1}[z(2 \pi)]=E_{1}[z(0)]$, but $E_{2,3}[z(2 \pi)]=-E_{2,3}[z(0)] \neq E_{2,3}[z(0)]$. Therefore, unlike $\lambda_{1}^{\alpha}[z(0)]$, the eigenstates $\lambda_{2,3}^{\alpha}[z(0)]$ are not adiabatic cyclic states of period $2 \pi{ }^{6}$

In general, the parameter space $\mathcal{M}$ consists of up to $N$ connected components; $\mathcal{M}$ $=\mathcal{M}_{1} \sqcup \mathcal{M}_{2} \sqcup \cdots \sqcup \mathcal{M}_{K}$, where $K \in I_{N}$ and $\mathcal{M}_{1}, \mathcal{M}_{2}, \ldots, \mathcal{M}_{K}$ are connected, mutually disjoint, and generally distinct covering spaces of $M$. For each $j \in I_{N}$, there is at least one $J \in I_{K}$ such that $E_{j}$ is a single-valued function on $\mathcal{M}_{J}$. In other words, given an initial eigenstate $\lambda_{j}$, one can choose a corresponding connected component $\mathcal{M}_{J}$ of $\mathcal{M}$ and use it as the parameter space for the corre-

${ }^{6}$ Here $\alpha=1$ if we use the open cover (15)-(17) of $M$. 
sponding geometric phase problem. This construction has the disadvantage that it makes the parameter space depend on the spectral label $j$ associated with the initial state. If we choose $\mathcal{M}$ as the parameter space for the problem, we should note that given a closed curve in $\mathcal{M}$, there may be initial eigenstates that are not cyclic along this curve. To avoid these complications we shall next offer an alternative construction for a parameter space that is more universal in the sense that along every closed curve in this space, all the initial eigenstates undergo adiabatic cyclic evolutions. These are the very properties of the parameter space used in Simon's ${ }^{10}$ holonomy interpretation of the adiabatic geometric phase for Hermitian Hamiltonians. ${ }^{1}$

First, we construct a principle $\mathfrak{S}_{N}$-bundle over $M$. Let $\left\{\left(U_{\alpha}, \phi_{\alpha}\right)\right\}_{\alpha \in \mathfrak{I}}$ and $\mathfrak{g}_{\alpha \beta}$ be, respectively, the family of local trivializations and the corresponding transition functions that we employed in the construction of $\mathcal{M}$. For each $\alpha, \beta \in \mathfrak{I}$ fulfilling $U_{\alpha} \cap U_{\beta} \neq \varnothing, R \in U_{\alpha} \cap U_{\beta}$, and $\mathfrak{g} \in \mathfrak{S}_{N}$, we identify $(R, \mathfrak{g}) \in U_{\alpha} \times \mathfrak{S}_{N}$ with $\left(R, \mathfrak{g}_{\beta \alpha}(R) \mathfrak{g}\right) \in U_{\beta} \times \mathfrak{S}_{N}$. This defines a principle $\mathfrak{S}_{N}$-bundle over $M$ that we denote by $\tilde{\mathfrak{M}}$. By construction, $\mathcal{M}$ is an associated fiber bundle for $\tilde{\mathfrak{M}} .{ }^{37} \tilde{\mathfrak{M}}$ is indeed an $N$ !-fold covering space of $M$ over which the eigenvalues $E_{j}$ are single-valued functions and the eigenstates undergo adiabatic cyclic evolutions along every closed curve $\widetilde{\mathfrak{C}}:[0, T] \rightarrow \widetilde{\mathfrak{M}}$. However, similarly to $\mathcal{M}, \widetilde{\mathfrak{M}}$ may generally be disconnected. Whenever the latter is the case, we can find a family of local trivializations of $\tilde{\mathfrak{M}}$ whose transition functions belong to a proper subgroup $\mathfrak{H}$ of the permutation group $\mathfrak{S}_{N}$, i.e., the structure group $\mathfrak{S}_{N}$ is reducible to $\mathfrak{H} .{ }^{37}$ This in turn enables us to reduce $\widetilde{\mathfrak{M}}$ to a principle $\mathfrak{H}$-bundle over $M$. Choosing the smallest subgroup $\mathfrak{H}$ of $\mathfrak{S}_{N}$ that allows for the reduction of $\tilde{\mathfrak{M}}$, we find an irreducible principle $\mathfrak{H}$-bundle over $M$ that we label as $\mathfrak{M}$. This is a connected $|\mathfrak{H}|$-fold $^{7}$ covering space of $M$ on which all the eigenvalues of the Hamiltonian are single-valued.

Computing $\tilde{\mathfrak{M}}$ and $\mathfrak{M}$ for the parameter-dependent Hamiltonian (13), we find that they are (diffeomorphic) copies of the manifold $\mathcal{M}$ that we obtained above. In contrast, for the Hamiltonian (18), $\mathfrak{M}=\mathcal{M}_{2,3} \varsubsetneqq \mathcal{M}$ and $\tilde{\mathfrak{M}}=\mathfrak{M} \times I_{2}$ (up to diffeomorphisms).

If the parameter-dependent Hamiltonian $H[R]$ is Hermitian for all $R \in M$, the eigenvalues are single-valued functions on $M$ and all the fiber bundles we have constructed are trivial. In particular, up to diffeomorphisms, we have $\mathcal{M}=M \times I_{N}, \tilde{\mathfrak{M}}=M \times \mathfrak{S}_{N}$, and $\mathfrak{M}=M$. The latter shows that $\mathfrak{M}$ reduces to the parameter space for the adiabatic geometric phases associated with the Hermitian Hamiltonians. ${ }^{1,10}$ The above properties of $\mathfrak{M}$ make it into the desired parameter space for general, possibly non-Hermitian matrix Hamiltonians.

\section{CONSTRUCTION OF THE BERRY-SIMON LINE BUNDLE}

In this section we examine the adiabatic geometric phase corresponding to the adiabatic cyclic evolution of the eigenstates of the initial Hamiltonian along an arbitrary closed curve $\mathfrak{C}:[0, T]$ $\rightarrow \mathfrak{M}$ in the parameter space $\mathfrak{M}^{8}$ Although the eigenstates $\lambda_{n}$ are single-valued smooth functions on $\mathfrak{M}$, the eigenvectors $\left|\psi_{n}[\cdot]\right\rangle$ can generally be defined as smooth single-valued functions on certain open subsets of $\mathfrak{M}$. Let $\left\{\mathcal{U}_{\mathfrak{a}}\right\}_{\mathfrak{a} \in \mathfrak{A}}$ be an open cover of $\mathfrak{M}$ such that $\mathcal{U}_{\mathfrak{a}}$ are diffeomorphic to $\mathbb{R}^{d}$ and $\left|\psi_{n}[\cdot]\right\rangle$ be a smooth single-valued function on $\mathcal{U}_{\mathfrak{a}}$ for all $\mathfrak{a} \in \mathfrak{A}$. We shall use $\mathcal{R}$ $:=\left(\mathcal{R}_{1}, \mathcal{R}_{2}, \ldots, \mathcal{R}_{d}\right)$ to denote the local coordinate representation of the points of $\mathfrak{M}$ that lie in $\mathcal{U}_{\mathfrak{a}}$ and use the superscript $\mathfrak{a}, \mathfrak{b}, \ldots$ to denote the local values of the relevant quantities at the points belonging to $\mathcal{U}_{\mathfrak{a}}$, e.g., we denote by $\left|\psi_{n}^{\mathrm{a}}[\mathcal{R}(t)]\right\rangle$ the value of $\left|\psi_{n}^{a}[\mathfrak{C}(t)]\right\rangle$ for all $t \in[0, T]$ such that $\mathfrak{C}(t) \in \mathcal{U}_{\mathfrak{a}}$.

Suppose for simplicity that $\mathfrak{C}$ lies entirely in $\mathcal{U}_{\mathfrak{a}}$ for some $\mathfrak{a} \in \mathfrak{A}$, in which case we can suppress the coordinate patch label $\mathfrak{a}$, and consider solving the Schrödinger equation [Eq. (8)] with the initial condition

\footnotetext{
${ }^{7}$ For a finite set $S$, we use $|S|$ to denote its order.

${ }^{8}$ Without loss of generality we can replace $\mathfrak{M}$ with $\tilde{\mathfrak{M}}$ or the connected component of $\mathcal{M}$ over which the eigenvalue $E_{n}$ is single-valued.
} 


$$
|\Psi(t=0)\rangle=k\left|\psi_{n}[\mathcal{R}(0)]\right\rangle,
$$

where $k \in \mathrm{C}-\{0\}$ is an arbitrary constant. If we employ adiabatic approximation, we can express the evolving state vector as

$$
|\Psi(t)\rangle=k e^{i\left[\delta_{n}(t)+\gamma_{n}(t)\right]}\left|\psi_{n}[\mathcal{R}(t)]\right\rangle,
$$

where

$$
\delta_{n}(t):=-\int_{0}^{t} E_{n}(\tau) d \tau, \quad \gamma_{n}(t):=\int_{0}^{t} i\left\langle\phi_{n}(\tau)\left|\frac{d}{d \tau}\right| \psi_{n}(\tau)\right\rangle d \tau,
$$

$E_{n}(t):=E_{n}[\mathcal{R}(t)],\left|\psi_{n}(t)\right\rangle:=\left|\psi_{n}[\mathcal{R}(t)]\right\rangle$, and $\left|\phi_{n}(t)\right\rangle:=\left|\phi_{n}[\mathcal{R}(t)]\right\rangle . \delta_{n}(T)$ and $\gamma_{n}(T)$ are the dynamical and geometric phase angles associated with the cyclic evolution of the initial eigenstate $\lambda_{n}[\mathcal{R}(0)]$.

Since $\mathfrak{M}$ is a covering space of $M$, the local properties of the eigenvectors, eigenvalues, and eigenstates of $H$ as functions on $\mathfrak{M}$ are identical with their local properties as functions on $M$. This allows for a straightforward generalization of the Berry-Simon complex line bundle ${ }^{1,10}$ for nonHermitian Hamiltonians. This is the spectral line bundle $L^{n}$ over the parameter space $\mathfrak{M}$ whose fiber over $\mathcal{R} \in \mathfrak{M}$ is the eigenstate $\lambda_{n}[\mathcal{R}] \subseteq \mathcal{H}$. We can construct it as follows.

For each $\mathfrak{a}, \mathfrak{b} \in \mathfrak{A}$ for which $\mathcal{U}_{\mathfrak{a}} \cap \mathcal{U}_{\mathfrak{b}} \neq \varnothing$ and for all $\mathcal{R} \in \mathcal{U}_{\mathfrak{a}} \cap \mathcal{U}_{\mathfrak{b}}$, both $\left|\psi_{n}^{\mathfrak{a}}[\mathcal{R}]\right\rangle$ and $\left|\psi_{n}^{b}[\mathcal{R}]\right\rangle$ belong to $\left.\lambda_{n}[\mathcal{R}]\right\rangle$. Therefore, there must exist $\mathcal{G}_{\mathfrak{a b}}: \mathcal{U}_{\mathfrak{a}} \cap \mathcal{U}_{\mathfrak{b}} \rightarrow G L(1, \mathrm{C})$ such that

$$
\left|\psi_{n}^{\mathrm{a}}[\mathcal{R}]\right\rangle=\mathcal{G}_{\mathfrak{a b}}(\mathcal{R})\left|\psi_{n}^{\mathrm{b}}[\mathcal{R}]\right\rangle .
$$

The line bundle $L^{n}$ is obtained by identifying $\left(\mathcal{R}, z^{\mathfrak{a}}\right) \in \mathcal{U}_{\mathfrak{a}} \times \mathrm{C}$ with $\left(\mathcal{R}, \mathcal{G}_{\mathfrak{b a}}(\mathcal{R}) z^{\mathfrak{a}}\right) \in \mathcal{U}_{\mathfrak{b}} \times \mathrm{C}$ for all $\mathcal{R} \in \mathcal{U}_{\mathfrak{a}} \cap \mathcal{U}_{\mathfrak{b}}$ and $z^{\mathfrak{a}} \in \mathrm{C}$. Let $\mathfrak{P}: L^{n} \rightarrow \mathfrak{M}$ denote the projection map for $L^{n}$ and, for each $\mathfrak{a} \in \mathfrak{A}$, $\varphi_{\mathfrak{a}}: \mathfrak{P}^{-1}\left(\mathcal{U}_{\mathfrak{a}}\right) \rightarrow \mathcal{U}_{\mathfrak{a}} \times \mathbb{C}$ be defined by $\varphi_{\mathfrak{a}}(\mathfrak{p}):=\left(\mathfrak{P}(\mathfrak{p}), z^{\mathfrak{a}}\right)$ for all $\mathfrak{p} \in \mathfrak{P}^{-1}\left(\mathcal{U}_{\mathfrak{a}}\right)$. Then $\left\{\left(\mathcal{U}_{\mathfrak{a}}, \varphi_{\mathfrak{a}}\right)\right\}_{\mathfrak{a} \in \mathfrak{A}}$ is a family of local trivializations of $L^{n}$ with transition functions $\mathcal{G}_{\mathfrak{a b}}$. This completes the construction of $L^{n}$ as a topological line bundle. Next, we endow this line bundle with a geometric structure, namely, Berry's connection one-form whose local expression is as follows:

$$
\mathcal{A}_{n}^{\mathfrak{a}}(\mathcal{R}):=i \sum_{j=1}^{d}\left\langle\phi_{n}^{\mathrm{a}}[\mathcal{R}]\left|\frac{\partial}{\partial \mathcal{R}^{j}}\right| \psi_{n}^{\mathrm{a}}[\mathcal{R}]\right\rangle d \mathcal{R}^{j}=i\left\langle\phi_{n}^{\mathrm{a}}[\mathcal{R}]|d| \psi_{n}^{\mathrm{a}}[\mathcal{R}]\right\rangle .
$$

With this choice for the geometry of $L^{n}$, the geometric phase factor $e^{i \gamma_{n}(T)}$ is identified with the holonomy associated with the curve $\mathfrak{C}$,

$$
e^{i \gamma_{n}(T)}=e^{i \oint_{\mathfrak{C}} \mathcal{A}_{n}^{\mathfrak{a}}}
$$

As shown in Appendix B, this construction applies also to the cases where $\mathfrak{C}$ does not lie in a single patch $\mathcal{U}_{\mathfrak{a}}$.

We end this section with three remarks.

(1) Whenever the Hamiltonian is non-Hermitian, the geometric phase factor (24) is not necessarily unimodular. This is consistent with the fact that in general under a nonunitary evolution, the norm of the evolving state vector changes.

(2) For cases where $H[\mathcal{R}]$ is Hermitian for all $\mathcal{R} \in \mathfrak{M}$, we can (locally) find an orthonormal set of eigenvectors $\left|\epsilon_{n}^{\mathrm{a}}[\mathcal{R}]\right\rangle$ of $H[\mathcal{R}]$ and a set of nowhere vanishing functions $\kappa_{n}^{\mathfrak{a}}: \mathcal{U}_{\mathfrak{a}} \rightarrow \mathrm{C}$ such that $\left|\psi_{n}^{\mathrm{a}}[\mathcal{R}]\right\rangle=\kappa_{n}^{\mathrm{a}}[\mathcal{R}]\left|\epsilon_{n}^{\mathrm{a}}[\mathcal{R}]\right\rangle,\left|\phi_{n}^{\mathrm{a}}[\mathcal{R}]\right\rangle=\kappa_{n}^{\mathrm{a}}[\mathcal{R}]^{-1 *}\left|\epsilon_{n}^{\mathrm{a}}[\mathcal{R}]\right\rangle$, and the Berry's connection oneform (23) takes the form

$$
\mathcal{A}_{n}^{\mathfrak{a}}(\mathcal{R})=i\left\langle\epsilon_{n}^{\mathfrak{a}}[\mathcal{R}]|d| \epsilon_{n}^{\mathrm{a}}[\mathcal{R}]\right\rangle+i d \ln \left(\kappa_{n}^{\mathfrak{a}}[\mathcal{R}]\right) .
$$

\footnotetext{
${ }^{9}$ In this construction we can relax the condition that $\mathcal{U}_{\mathfrak{a}}$ are diffeomorphic to $\mathbb{R}^{d}$. We can work with any open cover $\left\{\mathcal{U}_{\mathfrak{a}}\right\}_{\mathfrak{a} \in \mathfrak{A}}$ of $\mathfrak{M}$ such that each $\mathcal{U}_{\mathfrak{a}}$ admits an open cover $\left\{\mathfrak{u}_{\beta}^{\mathfrak{a}}\right\}_{\beta \in \mathfrak{B}}$ with $\mathfrak{u}_{\beta}^{\mathfrak{a}}$ diffeomorphic to $\mathbb{R}^{d}$ for all $\beta \in \mathfrak{B}$.
} 
The imaginary part of the first term on the right-hand side of this equation vanishes identically,

$$
\Im\left\{i\left\langle\epsilon_{n}^{\mathrm{a}}[\mathcal{R}]|d| \epsilon_{n}^{\mathrm{a}}[\mathcal{R}]\right\rangle\right\}=\frac{1}{2 i}\left(i\left\langle\epsilon_{n}^{\mathrm{a}}[\mathcal{R}] \mid d \epsilon_{n}^{\mathrm{a}}[\mathcal{R}]\right\rangle-\left\{i\left\langle\epsilon_{n}^{\mathrm{a}}[\mathcal{R}] \mid d \epsilon_{n}^{\mathrm{a}}[\mathcal{R}]\right\rangle\right\}^{*}\right)=\frac{1}{2} d\left\{\left\langle\epsilon_{n}^{\mathrm{a}}[\mathcal{R}] \mid \epsilon_{n}^{\mathrm{a}}[\mathcal{R}]\right\rangle\right\}=0 .
$$

This shows that for Hermitian Hamiltonians, the imaginary part of Berry's connection oneform (23) is an exact form (pure gauge). Therefore, it does not contribute to the geometric phase factor (24). This is consistent with the fact that for Hermitian Hamiltonians, the adiabatic geometric phase is a genuine (unimodular) phase factor.

(3) Similarly to the case of Hermitian Hamiltonians, we can compute the local curvature twoform $F_{n}$ associated with Berry's connection one-form (23). By definition,

$$
F_{n}(\mathcal{R}):=d \mathcal{A}_{n}^{\mathfrak{a}}(\mathcal{R})=i\left\langle d \phi_{n}^{\mathfrak{a}}[\mathcal{R}]|\wedge| d \psi_{n}^{\mathfrak{a}}[\mathcal{R}]\right\rangle .
$$

It is easy to check using (3) that $F_{n}(\mathcal{R})$ is invariant under changes of local eigenvectors (gauge transformations). Following essentially the same analysis as the one given in Ref. 1 for Hermitian Hamiltonians, we can put $F_{n}(\mathcal{R})$ in the following manifestly gauge-invariant form: ${ }^{38,39}$

$$
F_{n}(\mathcal{R})=i \sum_{m \neq n} \frac{\left\langle\phi_{n}^{\mathrm{a}}[\mathcal{R}]|d H[\mathcal{R}]| \psi_{m}^{\mathrm{a}}[\mathcal{R}]\right\rangle \wedge\left\langle\phi_{m}^{\mathrm{a}}[\mathcal{R}]|d H[\mathcal{R}]| \psi_{n}^{\mathrm{a}}[\mathcal{R}]\right\rangle}{\left(E_{m}[\mathcal{R}]-E_{n}[\mathcal{R}]\right)^{2}} .
$$

This expression is also invariant under changes of local trivialization. Therefore, it defines $F_{n}$ as a globally single-valued two-form on $\mathfrak{M}$.

\section{ADIABATIC GEOMETRIC PHASE FOR GENERAL $2 \times 2$ HAMILTONIANS}

$2 \times 2$ matrix Hamiltonians provide the simplest nontrivial toy models in the study of geometric phases. As far as the physical (measurable) quantities are concerned, we can confine our attention to traceless $2 \times 2$ Hamiltonians ${ }^{10}$

$$
H=\left(\begin{array}{cc}
a & b \\
c & -a
\end{array}\right)
$$

where $a, b, c \in \mathrm{C}$. It is not difficult to show that the parameter space $M$ for these models is given by

$$
M:=\left\{a, b, c \in \mathbb{C} \mid a^{2}+b c \neq 0\right\} \varsubsetneqq \mathrm{C}^{3}=\mathbb{R}^{6} .
$$

It is a real six-dimensional smooth manifold.

$H$ is a single-valued function on $M$ with eigenvalues ${ }^{11}$

$$
E_{ \pm}= \pm f, \quad f:=\sqrt{a^{2}+b c} .
$$

Because of the appearance of the square root (with a generally complex argument) in the expression for $f, E_{ \pm}$are, in general, only locally single-valued functions on $M$. The parameter space $\mathfrak{M}$, which happens to coincide with $\mathcal{M}$ and $\tilde{\mathfrak{M}}$, is a double covering space of $M$ over which $f$ is a single-valued function. This implies that each point of $\mathfrak{M}$ is uniquely determined by the values of $a, b, c$, and $f$. Note that $a, b$, and $c$ determine $f$ up to a sign. Specifying the value of $f$ corresponds to fixing the sign ambiguity. In the following we shall use $\mathcal{R}$ to denote the local coordinates of the points of $\mathfrak{M} ; \mathcal{R}$ is determined by the values of $a, b, c$, and $f$.

\footnotetext{
${ }^{10}$ One can transform the set of all non-Hermitian $2 \times 2$ Hamiltonians to a set of traceless $2 \times 2$ non-Hermitian Hamiltonians by canonical transformations that leave all the physical quantities of the system, including geometric phases, invariant. ${ }^{45,46}$

${ }^{11}$ We use \pm for the spectral label $n$.
} 
We can view the eigenvectors $\left|\psi_{ \pm}[\cdot]\right\rangle$ of $H$ as global sections of the line bundles $L^{ \pm}$. It turns out that these fail to be continuous global sections and that we can only construct smooth local sections corresponding to $\left|\psi_{ \pm}[\cdot]\right\rangle$. This requires constructing an open cover of $\mathfrak{M}$. To achieve this, we introduce

$$
\mathcal{P}^{ \pm}:=\{\mathcal{R} \in \mathfrak{M} \mid b c=0, \quad f= \pm a \neq 0\},
$$

which are a pair of disjoint closed subsets ${ }^{12}$ of $\mathfrak{M}$, and consider their complement in $\mathfrak{M}$, namely,

$$
\mathfrak{M}^{1}:=\mathfrak{M}-\mathcal{P}^{-}, \quad \mathfrak{M}^{2}:=\mathfrak{M}-\mathcal{P}^{+} .
$$

Clearly these are open subsets of $\mathfrak{M}$ satisfying $\mathfrak{M}^{1} \cup \mathfrak{M}^{2}=\mathfrak{M}$. Hence $\left\{\mathfrak{M}^{1}, \mathfrak{M}^{2}\right\}$ is an open cover of $\mathfrak{M}$.

Now, we are in a position to define a set of eigenvectors of the Hamiltonian (25) that yields smooth local sections of the bundle $L^{ \pm}$. These are the functions $\left|\psi_{ \pm}^{\nu}[\cdot]\right\rangle: \mathfrak{M}^{\nu} \rightarrow L^{ \pm}$, with $\nu \in I_{2}$, that are defined by

$$
\left|\psi_{+}^{1}[\mathcal{R}]\right\rangle:=\left(\begin{array}{c}
f+a \\
c
\end{array}\right), \quad\left|\psi_{-}^{1}[\mathcal{R}]\right\rangle:=\left(\begin{array}{c}
-b \\
f+a
\end{array}\right), \quad\left|\psi_{ \pm}^{2}[\mathcal{R}]\right\rangle:=\left.\left|\psi_{\mp}^{1}[\mathcal{R}]\right\rangle\right|_{f \rightarrow-f} .
$$

We can also construct the corresponding left eigenvectors $\left|\phi_{ \pm}^{\nu}[\mathcal{R}]\right\rangle$ of the Hamiltonian that together with $\left|\psi_{ \pm}^{\nu}[\mathcal{R}]\right\rangle$ for a biorthonormal system for the Hilbert space,

$$
\left|\phi_{+}^{1}[\mathcal{R}]\right\rangle=\frac{1}{2 f^{*}\left(f^{*}+a^{*}\right)}\left(\begin{array}{c}
f^{*}+a^{*} \\
b^{*}
\end{array}\right), \quad\left|\phi_{-}^{1}[\mathcal{R}]\right\rangle=\frac{1}{2 f^{*}\left(f^{*}+a^{*}\right)}\left(\begin{array}{c}
-c^{*} \\
f^{*}+a^{*}
\end{array}\right), \quad\left|\phi_{ \pm}^{2}[\mathcal{R}]\right\rangle:=\left.\left|\phi_{\mp}^{1}[\mathcal{R}]\right\rangle\right|_{f \rightarrow-f} .
$$

Next, we use (30) and (31) to give local expressions for Berry's connection one-form on $L^{ \pm}$. Using the superscript $\nu$ to emphasize that the domain of definition of the corresponding connection one-form is $\mathfrak{M}^{\nu}$, we have

$$
\begin{gathered}
\mathcal{A}_{+}^{1}(\mathcal{R})=\frac{i}{2 f}\left(\frac{b d c}{f+a}+d f+d a\right), \quad \mathcal{A}_{-}^{1}(\mathcal{R})=\frac{i}{2 f}\left(\frac{c d b}{f+a}+d f+d a\right), \\
\mathcal{A}_{ \pm}^{2}(\mathcal{R})=\left.\mathcal{A}_{+}^{1}(\mathcal{R})\right|_{f \rightarrow-f} .
\end{gathered}
$$

For a closed curve $\mathfrak{C}:[0, T] \rightarrow \mathfrak{M}$ that lies entirely in $\mathfrak{M}^{1}$ or $\mathfrak{M}^{2}$, we can use (24) and either of (32) or (33) to calculate the corresponding adiabatic geometric phase. If $\mathfrak{C}$ is not contained in $\mathfrak{M}^{1}$ or $\mathfrak{M}^{2}$, we must compute the contributions to the geometric phase from segments of $\mathfrak{C}$ that lie in $\mathfrak{M}^{1}$ and $\mathfrak{M}^{2}$ and use the transition functions $\mathcal{G}_{2,1}^{ \pm}: \mathfrak{M}^{1} \cap \mathfrak{M}^{2} \rightarrow G L(1, \mathrm{C})$ to patch up these contributions and obtain the total geometric phase. We give the details of this well-known construction in Appendix B. Here we only include the expression for the transition functions that we obtained using (22) and (30),

$$
\mathcal{G}_{2,1}^{+}(\mathcal{R})=\frac{-b}{f+a}, \quad \mathcal{G}_{2,1}^{-}(\mathcal{R})=\frac{c}{f+a}, \quad \forall \mathcal{R} \in \mathfrak{M}^{1} \cap \mathfrak{M}^{2} .
$$

In the remainder of this section we examine a number of specific examples. These will in particular be helpful in our discussion of the geometrical versus topological nature of EP-related geometric phases.

\footnotetext{
${ }^{12}$ Note that on $\mathfrak{M}, f \neq 0$. This is the reason why $\mathcal{P}^{ \pm}$are closed subsets of $\mathfrak{M}$.
} 


\section{A. Two symmetric examples}

In this subsection we determine the parameter space $\mathfrak{M}$ for two symmetric matrix Hamiltonians whose EPs are branch points of the function $f$ over the parameter space $M$. We also give the adiabatic geometric phase for curves enclosing the EPs.

(a) Consider the symmetric Hamiltonian

$$
H[z]:=\left(\begin{array}{cc}
1+z & i(1-z) \\
i(1-z) & -(1+z)
\end{array}\right), \quad z \in M:=\mathrm{C}-\{0\},
$$

for which $f=2 \sqrt{z}$. The parameter space $\mathfrak{M}$ is a double covering space of $M$ over which $f$ is single valued. Clearly, it is the two-sheeted Riemann surface over the punctured complex plane which we described in Sec. II. The point $z=0$ is the only EP for this Hamiltonian.

(b) Let

$$
H[z]:=\left(\begin{array}{cc}
1+z & 1-z \\
1-z & -(1+z)
\end{array}\right), \quad z \in M:=\mathrm{C}-\{-i,+i\} .
$$

Then $f=2 \sqrt{\left(1+z^{2}\right)}=2 \sqrt{(z+i)(z-i)}$ and $\mathfrak{M}$ is the Riemann surface with two branched points.

For both of these examples, the Hamiltonian is symmetric, Berry's connection one-form is flat, and consequently the geometric phase factor associated with any closed curve encircling an EP has the topological value of $\pm 1 .{ }^{12}$

\section{B. Two nonsymmetric examples}

Here we consider a pair of nonsymmetric Hamiltonians depending also on a single complex parameter and examine the adiabatic geometrical phase problem for closed curves enclosing EPs.

(a) For the Hamiltonian

$$
H[z]:=\left(\begin{array}{cc}
z & 1 \\
0 & -z
\end{array}\right), \quad z \in M:=\mathrm{C}-\{0\},
$$

we have $a=f=z, b=1$, and $c=0$. Therefore, $z=0$ is an EP, $\tilde{\mathfrak{M}}=\mathcal{M}=\{-1,+1\} \times M, \mathfrak{M}=M$, and in view of Eqs. (30)-(33),

$$
\mathcal{A}_{+}(\mathcal{R})=0, \quad \mathcal{A}_{-}(\mathcal{R})=\frac{i d z}{z} .
$$

This, together with (24), yields the value of 0 for the adiabatic geometric phase angle corresponding to every closed curve in $\mathfrak{M}$ including those encircling the EP.

(b) Consider the following family of Hamiltonians:

$$
H[z]=\left(\begin{array}{cc}
\alpha z & 1 \\
\left(\beta^{2}-\alpha^{2}\right) z^{2} & -\alpha z
\end{array}\right),
$$

where we treat $\alpha$ and $\beta$ as fixed but arbitrary nonzero complex constants and $z$ as the physical parameter that is made time dependent. Then $z=0$ is the only EP, $M:=\mathrm{C}-\{0\}, f$ $=\beta z, \mathfrak{M}=M$, and in light of (30)-(33),

$$
\mathcal{A}_{+}(\mathcal{R})=i \frac{d z}{z}\left(\frac{3 \beta-\alpha}{2 \beta}\right), \quad \mathcal{A}_{-}(\mathcal{R})=i \frac{d z}{z}\left(\frac{\alpha+\beta}{2 \beta}\right) .
$$

For this system the adiabatic geometric phase angles $\gamma_{ \pm}(T)$ associated with the curve $\mathfrak{C}(t)$ $:=e^{2 \pi i t / T}$ are given (up to integer multiples of $2 \pi$ ) by 


$$
\gamma_{+}(T)=-\pi\left(1-\frac{\alpha}{\beta}\right), \quad \gamma_{-}(T)=-\pi\left(1+\frac{\alpha}{\beta}\right) .
$$

Note that $\mathfrak{C}$ does enclose the EP, but depending on the choice of $\alpha$ and $\beta, \gamma_{ \pm}(T)$ can take arbitrary values. This seems to contradict the results of Ref. 12 where the adiabatic geometric phase angles for general $2 \times 2$ Hamiltonians are claimed to take the values $\pm \pi$.

\section{A three-parameter family of Hamiltonians}

In Ref. 14, the authors considered traceless $2 \times 2$ Hamiltonians with a constant non-Hermitian part, i.e., $H[R]=(\vec{R}-(i / 2) \vec{\Gamma}) \cdot \vec{\sigma}$, where $\vec{R}:=R=\left(R_{1}, R_{2}, R_{3}\right) \in \mathbb{R}^{3}$ are the physical parameters of the system, $\vec{\Gamma}=\left(\Gamma_{1}, \Gamma_{2}, \Gamma_{3}\right) \in \mathbb{R}^{3}-\{\overrightarrow{0}\}$ is a constant nonzero vector, $\vec{\sigma}:=\left(\sigma_{1}, \sigma_{2}, \sigma_{3}\right)$, and $\sigma_{1}, \sigma_{2}, \sigma_{3}$ are Pauli matrices. Because we can transform the anti-Hermitian part of $H[R]$, namely, $(i / 2) \vec{\Gamma} \cdot \vec{\sigma}$, to a diagonal matrix by performing a constant similarity transformation, we can confine our attention to the case $\vec{\Gamma}=\left(0,0, \Gamma_{3}\right)$ without losing generality. This leads to the following explicit form for $H[R]$ :

$$
H[R]=\left(\begin{array}{cc}
R_{3}-\frac{i \Gamma}{2} & R_{1}-i R_{2} \\
R_{1}+i R_{2} & -\left(R_{3}-\frac{i \Gamma}{2}\right)
\end{array}\right),
$$

where we dropped the label 3 in $\Gamma_{3}$. It is easy to see that $f=\sqrt{|\vec{R}|^{2}-i R_{3} \Gamma-\Gamma^{2} / 4}$. Therefore, there is an infinity of EPs forming the circle,

$$
S^{1}:=\left\{\left(R_{1}, R_{2}, R_{3}\right) \in \mathbb{R}^{3} \mid R_{1}^{2}+R_{2}^{2}=\frac{\Gamma^{2}}{4}, \quad R_{3}=0\right\} .
$$

This implies that $M:=\mathbb{R}^{3}-S^{1}$ and $\mathfrak{M}$ is a double covering space of $M$ over which $f$ is single valued.

The authors of Refs. 14 and 27 calculated the geometric phase for closed curves in $M$ (not $\mathfrak{M}$ ) that encircle or approach $S^{1}$. If one changes the parameters along the noncontractible curves $C$ in $M$ (by traversing it once), the eigenstates of the Hamiltonian do not return to their initial value. Therefore, they do not undergo (adiabatic) cyclic evolutions. In the following, we examine the structure of the parameter space $\mathfrak{M}$ for this model.

We can simplify the situation by considering the subfamily of (36) obtained by setting $R_{2}$ $=0$. Then $f=\sqrt{R_{1}^{2}+R_{3}^{2}-i R_{3} \Gamma-\Gamma^{2} / 4}$, the Hamiltonian $H[R]$ [with $\left.R:=\left(R_{1}, R_{3}\right)\right]$ has a pair of EPs located at $R_{ \pm}:=\left(R_{1}= \pm \Gamma / 2, R_{3}=0\right)$, and $M=\mathbb{R}^{2}-\left\{R_{-}, R_{+}\right\}$.

Now, consider the closed curves $C_{ \pm}:[0, T] \rightarrow M$ defined by

$$
C_{ \pm}(t)=\left(R_{1}(t), R_{3}(t)\right)=\left( \pm\left(\frac{\Gamma}{2}+\epsilon \cos \omega t\right), \epsilon \sin \omega t\right), \quad \forall t \in[0, T],
$$

where $\omega:=2 \pi / T$ and $\epsilon$ is a positive real number less than $\Gamma / 2$. It is not difficult to see that $C_{ \pm}$ encloses the EP located at $R_{ \pm}$.

Along $C_{ \pm}$the value of $f$ changes according to

$$
f\left(C_{ \pm}(t)\right)=\sqrt{\epsilon\left(\epsilon+\Gamma e^{-i \omega t}\right)} .
$$

Hence $f\left(C_{ \pm}(T)\right)=-f\left(C_{ \pm}(0)\right)$. The same holds for every closed curve in $M$ that is homotopic to $C_{ \pm}$. Furthermore, because $f$ behaves similarly to the complex-valued function $h(z)$ $:=\sqrt{(z-\Gamma / 2)(z+\Gamma / 2)}$, the parameter space $\mathfrak{M}$, over which $f$ is single-valued, is diffeomorphic to the Riemman surface corresponding to $h(z)$ (see, for example, Ref. 40). To the closed curves $C_{ \pm}$ in $M$, one may associate the closed curves $\mathfrak{C}_{ \pm}$in $\mathfrak{M}$ that project under the bundle projection map 
$\pi: \mathfrak{M} \rightarrow M$ onto $\mathfrak{C}_{ \pm}$, and as one goes around $\mathfrak{C}_{ \pm}$once, one traverses $C_{ \pm}$twice. Under this change of parameters, the eigenstates of the initial Hamiltonian perform a cyclic adiabatic evolution, and according to the analysis of Ref. 12 the corresponding geometric phases are topological in nature. As we explain in Sec. IV D, this behavior stems from the choice of traversing $C_{ \pm}$twice.

\section{Are EP-related geometric phases topological?}

In Ref. 12, the authors calculated the adiabatic geometric phase around the EPs for the general $2 \times 2$ matrix Hamiltonians and found it to be topological, i.e., the geometric phase angle associated with each cycle equals $\pm \pi \cdot{ }^{13}$ In the following, we re-examine the argument leading to this conclusion and show that it is based on certain implicit assumptions that do not hold generally.

We begin our assessment of the above-mentioned result of Ref. 12 for nonsymmetric $2 \times 2$ Hamiltonians by noting that the authors of Ref. 12 work with the parameter space $M$ instead of $\mathfrak{M}$. Consequently, in order to make eigenstates undergo adiabatic cyclic evolutions, they consider encircling EPs along closed curves $C$ in $M$ twice. They use the notation $2 C$ to emphasize this point. This applies whenever the EP is a branch point. There are two shortcomings with this approach. First, there are cases where the enclosed EP is not a branch point. A simple example is the EP associated with the Hamiltonian (35). Second, and much more importantly, to obtain an adiabatic cyclic evolution of the eigenstates of the initial Hamiltonian, one does not need to traverse the same curve $C$ twice. One can alternatively go around $C$ once and then follow an arbitrary closed curve $C^{\prime}$ that does not enclose any EPs other than those enclosed by $C$. We denote the resulting combined curve by $C+C^{\prime}$, which yields $2 C$ if we choose $C^{\prime}=C$ (with the same orientation). More generally, we can choose (in place of $C+C^{\prime}$ ) an arbitrary smooth curve $\widetilde{C}$ in $M$ that is homotopic to $2 C$. The domain of the validity of the result of Ref. 12 pertaining the topological nature of the geometric phases is restricted to branch point EPs and the special choice $\widetilde{C}=2 C$. In general, for an EP which is a branch point there are an infinity of other choices for $\widetilde{C}$, and the resulting geometric phases are sensitive to its shape. Furthermore, whenever the EP is not a branch point, the cyclic evolution is generated by traversing a closed curve in $M$ once. In this case, one obtains the geometric phase of Garrison and Wright, ${ }^{9}$ which being a direct generalization of Berry's phase is generally geometrical and not topological.

\section{CONCLUSION}

If an eigenstate of the Hamiltonian is a single-valued function on a closed curve $C$ in the space $M$ of the parameters of the Hamiltonian, it undergoes an adiabatic cyclic evolution along $C$. In this case, one may pursue the approach of Garrison and Wright ${ }^{9}$ and define the non-Hermitian generalization of Berry's adiabatic geometric phase for this cyclic evolution. This is however not generally the case. If $C$ encircles an exceptional point, the eigenstate in question might not be single-valued along $C$. A careful description of this situation calls for a closer look at the appropriate parameter space for the problem of the adiabatic geometric phase for non-Hermitian Hamiltonians. In this article, we have constructed an appropriate parameter space $\mathfrak{M}$ for this problem that is a certain covering space of $M$. We have then introduced the non-Hermitian generalization of the Berry-Simon complex line bundle over $\mathfrak{M}$ whose holonomies coincide with the adiabatic geometric phases.

For $2 \times 2$ matrix Hamiltonians, we have offered explicit and general formulas for the adiabatic geometric phase corresponding to arbitrary closed curves in $\mathfrak{M}$ and showed that contrary to some recent claims, these phases are not generally topological in nature. In light of the developments reported in Refs. 11 and 24, it should be possible to confirm this prediction experimentally.

\footnotetext{
${ }^{13}$ This is in obvious conflict with the results of Sec. IV B.
} 


\section{ACKNOWLEDGMENTS}

H.M.-D. would like to express his gratitude to Mostafa Esfahanizadeh, Mohammad Reza Razvan, and Mohammad Khorrami for fruitful discussions and helpful comments.

\section{APPENDIX A: ALGEBRAIC TOPOLOGICAL PROPERTIES OF $\tilde{\mathfrak{M}}$}

In this appendix we use some well-known algebraic topological tools to explore certain properties of the parameter space $\tilde{\mathfrak{M}}$, which we constructed as a principal $\mathfrak{S}_{N}$-bundle over $M$ with projection map $\pi: \tilde{\mathfrak{M}} \rightarrow M$. We begin by recalling that each closed curve $C$ in $M$ that passes through a point $R \in M$ determines an element $[C]$ of the fundamental group $\pi_{1}(M, R)$ of $M$. Because $M$ is a connected manifold, we can omit the base point $R$ and use $\pi_{1}(M)$ for $\pi_{1}(M, R){ }^{41}$ We can define a right action of $C$ on the fiber $\pi^{-1}(R)$ over $R$, which maps every point $\mathcal{R}_{i}$ $\in \pi^{-1}(R)$ to the end point $\mathcal{R}_{i}$. $C \in \pi^{-1}(R)$ of the unique lift of $C$ to $\tilde{\mathfrak{M}}$ that has $\mathcal{R}_{i}$ as its initial point. Because $\tilde{\mathfrak{M}}$ is a covering space of $M$, homotopic curves in $M$ yield the same right action on $\pi^{-1}(R) .{ }^{42,43}$ Therefore, the above construction defines a right action of the fundamental group $\pi_{1}(M)$ on the fibers of $\tilde{\mathfrak{M}}$.

Next, consider the homomorphism $\pi_{*}: \pi_{1}\left(\tilde{\mathfrak{M}}, \mathcal{R}_{i}\right) \rightarrow \pi_{1}(M)$ induced by $\pi$ that maps the homotopy class $\left[\mathcal{C}_{i}\right]$ of the closed curves $\mathcal{C}_{i}$ passing through $\mathcal{R}_{i}$ in $\tilde{\mathfrak{M}}$ onto the homotopy class $[\pi$ ${ }^{\circ} \mathcal{C}_{i}$ ] of the projection of $\mathcal{C}_{i}$ under $\pi .{ }^{14}$ One can show that for every $\mathcal{R}_{i} \in \pi^{-1}(R)$, and every curve $C$ in $M$ whose homotopy class $[C]$ belongs to $\pi_{*}\left(\pi_{1}\left(\tilde{\mathfrak{M}}, \mathcal{R}_{i}\right)\right), \quad \mathcal{R}_{i} \cdot C=\mathcal{R}_{i}$. Therefore, $\pi_{*}\left(\pi_{1}\left(\tilde{\mathfrak{M}}, \mathcal{R}_{i}\right)\right)$ has a trivial action on $\pi^{-1}(R)$, i.e., with respect to its action on $\pi^{-1}(R)$, $\pi_{*}\left(\pi_{1}\left(\tilde{\mathfrak{M}}, \mathcal{R}_{i}\right)\right)$ is an isotropy subgroup of $\pi_{1}(M)$. This in turn implies that $\pi_{*}\left(\pi_{1}\left(\tilde{\mathfrak{M}}, \mathcal{R}_{j}\right)\right)$ is a normal subgroup of $\pi_{1}(M)$; for every $[C] \in \pi_{*}\left(\pi_{1}\left(\tilde{\mathfrak{M}}, \mathcal{R}_{j}\right)\right)$ and every $\left[C_{0}\right] \in \pi_{1}(M)$, we have $\mathcal{R}_{i} \cdot\left(C_{0} \cdot C \cdot C_{0}^{-1}\right)=\mathcal{R}_{i} \cdot C \in \tilde{\mathfrak{M}}$. We can use this observation to infer that $\pi_{*}\left(\pi_{1}\left(\tilde{\mathfrak{M}}, \mathcal{R}_{i}\right)\right)$ is independent of the choice of $\mathcal{R}_{i}$. Therefore, we shall use the abbreviated notation $\pi_{*}\left(\pi_{1}(\tilde{\mathfrak{M}})\right)$ for $\pi_{*}\left(\pi_{1}\left(\tilde{\mathfrak{M}}, \mathcal{R}_{i}\right)\right)$.

For the parameter space $\mathcal{M}$ which is also a covering space of $M$, it may happen that $\pi_{*}\left(\pi_{1}\left(\mathcal{M}, \mathcal{R}_{i}\right)\right)$ depends on $\mathcal{R}_{i}$ and $\pi_{*}\left(\pi_{1}\left(\mathcal{M}, \mathcal{R}_{i}\right)\right)$ fails to be an isotropy subgroup of $\pi_{1}(M)$. In this case there exist closed curves in $\mathcal{M}$ that do not yield a cyclic evolution for some of the eigenstates of the initial Hamiltonian.

It is possible for $\widetilde{\mathfrak{M}}$ to be a disconnected covering space of $M$. In this case, the right action of $\pi_{1}(M)$ on $\pi^{-1}(R)$ is not transitive. This means that we can choose the local trivializations of $\tilde{\mathfrak{M}}$ in such a way that the transition functions take their values in an irreducible proper subgroup $\mathfrak{H}$ of $\mathfrak{S}_{N}$. We will next denote by $\mathfrak{M}$ one of the connected components of $\tilde{\mathfrak{M}}$ and argue that $\mathfrak{M}$ and every other connected component of $\tilde{\mathfrak{M}}$ is diffeomorphic as manifolds with the principal $\mathfrak{H}$-bundle obtained by reducing $\tilde{\mathfrak{M}}$.

As shown in Ref. 42, $\pi^{-1}(R) \cap \mathfrak{M}$ is equivalent (bijective) to the quotient set $\pi_{1}(M) / \pi_{*}\left(\pi_{1}(\mathfrak{M})\right)$, and the order of the fibers of $\mathfrak{M}$ is equal to the order of $\pi_{1}(M) / \pi_{*}\left(\pi_{1}(\mathfrak{M})\right)$. Note that $\pi_{*}\left(\pi_{1}(\mathfrak{M})\right)$ is a normal subgroup of $\pi_{1}(M)$, and $\pi_{1}(M) / \pi_{*}\left(\pi_{1}(\mathfrak{M})\right)$ is a group isomorphic to $\mathfrak{H}$. Therefore, the order of fibers of $\mathfrak{M}$ is equal to the order $|\mathfrak{H}|$ of $\mathfrak{H}$. Now, consider arbitrary points $\mathcal{R}_{j}, \mathcal{R}_{k} \in \pi^{-1}(R)$ such that $\mathcal{R}_{j} \in \mathfrak{M}$ and $\mathcal{R}_{k} \notin \mathfrak{M}$. Since $\mathcal{R}_{k}$ is equivalent to an ordered set of eigenvalues of $H[R]$, the action of the group $\pi_{1}(M)$ on $\mathcal{R}_{k}$ is equivalent to its action on $\mathcal{R}_{j} \in \pi^{-1}(R) \cap \mathfrak{M}$. This suggests the existence of an $|\mathfrak{H}|$-fold covering space $\mathfrak{M}^{\prime}$ of $M$ that contains $\mathcal{R}_{k}$ and is isomorphic (as bundles) to $\mathfrak{M}$. As a result the parameter space $\tilde{\mathfrak{M}}$ consists of $N ! /|\mathfrak{H}|$ disjoint connected $|\mathfrak{H}|$-fold covering spaces of $M$ which are diffeomorphic to $\mathfrak{M}$.

\footnotetext{
${ }^{14}$ Note that given a connected covering space $\mathcal{N}$ of a manifold $N$, an arbitrary point $R \in N$, and any two points $\mathcal{R}_{i}$ and $\mathcal{R}_{j}$ of the fiber over $R, \pi_{*}\left(\pi_{1}\left(\mathcal{N}, \mathcal{R}_{i}\right)\right)$ and $\pi_{*}\left(\pi_{1}\left(\mathcal{N}, \mathcal{R}_{j}\right)\right)$ are conjugate subgroups of $\pi_{1}(N) .{ }^{42,43}$
} 


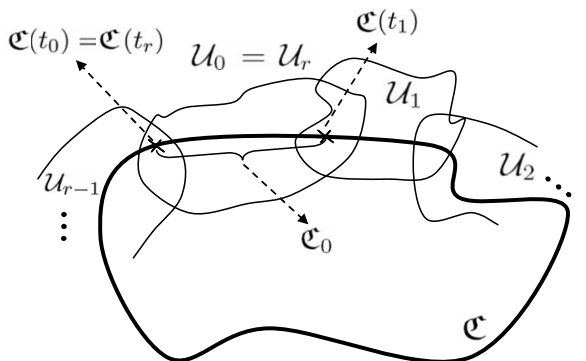

(a)

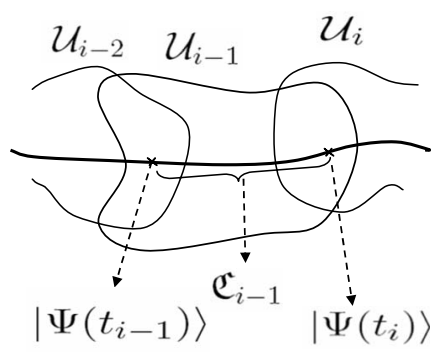

(b)

FIG. 1. (a) An arbitrary closed curve $\mathfrak{C}$ (the bold curve) visiting different open patches. (b) The $i-1$ th segment of the curve $\mathfrak{C}$, namely, $\mathfrak{C}_{i-1}$, with the initial and final points $\mathfrak{C}_{i-1}\left(t_{i-1}\right)$ and $\mathfrak{C}_{i-1}\left(t_{i}\right)$.

\section{APPENDIX B: ADIABATIC GEOMETRIC PHASE FOR A GENERAL CLOSED CURVE IN $\mathfrak{M}$}

First, consider a closed $\mathfrak{C}$ lying in an open patch $\mathcal{U}_{\mathfrak{a}}$ of $\mathfrak{M}$, so that we can suppress the patch label $\mathfrak{a}$ in our calculations. Then, given an adiabatic solution $|\Psi(t)\rangle$ of the Schrödinger equation [Eq. (8)] fulfilling the initial condition (19), the relation

$$
|\tilde{\Psi}[\mathcal{R}(t)]\rangle:=e^{-i \delta_{n}(t)}|\Psi(t)\rangle:=k e^{i \gamma_{n}(\mathfrak{C}(t))}\left|\psi_{n}[\mathcal{R}(t)]\right\rangle,
$$

defines a single-valued function $|\widetilde{\Psi}[\cdot]\rangle: \mathfrak{C} \rightarrow L^{n}$, which we identify with the horizontal lift of the curve $\mathfrak{C}^{1,37,41,44}$ As $t$ varies in the range $[0, T],|\widetilde{\Psi}[\mathcal{R}(t)]\rangle$ traces the parallel transport of $|\Psi(\mathcal{R}(0))\rangle$ along $\mathfrak{C}$. The phase factor $\exp \left(i \gamma_{n}(\mathfrak{C}(t))\right)$ appearing in (B1) is not gauge-invariant unless $\mathcal{R}(t)$ $=\mathcal{R}(0)$. By construction, the latter holds for $t=T$. The phase factor $\exp \left(i \gamma_{n}(\mathfrak{C}(T))\right)$ is the holonomy of the closed curve $\mathfrak{C}$.

Next, we wish to generalize the expression for $\exp \left(i \gamma_{n}(\mathfrak{C}(T))\right)$ to the cases that $\mathfrak{C}$ does not lie in a single open patch. This requires the examination of the behavior of Berry's connection one-form (23) under a change of local trivializations. In view of (22) and (23),

$$
\mathcal{A}_{n}^{\mathfrak{b}}(\mathcal{R})=\mathcal{A}_{n}^{\mathfrak{a}}(\mathcal{R})+i \frac{d \mathcal{G}_{n}^{\mathfrak{b}, \mathfrak{a}}(\mathcal{R})}{\mathcal{G}_{n}^{\mathfrak{b}, \mathfrak{a}}(\mathcal{R})}
$$

Suppose that $\mathfrak{C}$ is a closed curve lying in the union of the open patches $\mathcal{U}_{0}, \mathcal{U}_{1}, \mathcal{U}_{2}, \ldots, \mathcal{U}_{r-1}$, where $r \in \mathbb{Z}^{+}$[see Fig. 1(a) and footnote 9]. Let $\mathcal{U}_{r}:=\mathcal{U}_{0} ; t_{0}, t_{1}, t_{2}, \ldots, t_{r-1}, t_{r} \in[0, T]$ be such that $0=t_{0}$ $<t_{1}<t_{2}<\cdots<t_{r-1}<t_{r}=T$; for all $i \in\{1,2, \ldots, r\}, \mathfrak{C}\left(t_{i}\right) \in \mathcal{U}_{i-1} \cap \mathcal{U}_{i}, \mathfrak{C}\left(t_{0}\right):=\mathfrak{C}\left(t_{r}\right) \in \mathcal{U}_{r-1} \cap \mathcal{U}_{0}$ and for all $t \in\left[t_{i-1}, t_{i}\right]$, the point $\mathfrak{C}(t)$ belongs to $\mathcal{U}_{i-1}$. The latter determine the segments of $\mathfrak{C}$ belonging to $\mathcal{U}_{i-1}$ that we denote by $\mathfrak{C}_{i-1} \cdot{ }^{15}$ Next, let $\left|\psi_{n}^{i-1}[\cdot]\right\rangle: \mathcal{U}_{i-1} \rightarrow L^{n}$ be the smooth local section defined on $\mathcal{U}_{i-1}$.

For $t \in\left[t_{i-1}, t_{i}\right]$, the state vector $|\Psi(t)\rangle$ that evolves adiabatically according to the Schrödinger equation [Eq. (8)] with the initial condition $\left|\Psi\left(t_{i-1}\right)\right\rangle=k_{i-1}\left|\psi_{n}^{i-1}\left[\mathcal{R}\left(t_{i-1}\right)\right]\right\rangle$ satisfies

$$
\left|\Psi\left(t_{i}\right)\right\rangle=k_{i}\left|\psi_{n}^{i}\left[\mathcal{R}\left(t_{i}\right)\right]\right\rangle
$$

[see Fig. 1(b)]. Here the coefficient $k_{i}$ is given by

$$
k_{i}:=\mathcal{K}_{i} k_{i-1}, \quad \mathcal{K}_{i}:=e^{i\left(\delta_{n}^{i-1}+\gamma_{n}\left(\mathfrak{C}_{i-1}\right)\right)} \mathcal{G}_{n}^{i-1, i}\left(\mathcal{R}\left(t_{i}\right)\right), \quad k_{0}:=k,
$$

\footnotetext{
${ }^{15}$ It can occur that curve $\mathfrak{C}$ visits a given patch more than once. We account for this situation by allowing the possibility of using different labels for the same patch, i.e., $\mathcal{U}_{i}$ are not necessarily distinct patches.
} 


$$
\delta_{n}^{i-1}:=-\int_{t_{i-1}}^{t_{i}} E_{n}(t) d t, \quad \gamma_{n}\left(\mathfrak{C}_{i-1}\right):=\int_{\mathfrak{C}_{i-1}} \mathcal{A}_{n}^{i-1},
$$

and $\mathcal{G}_{n}^{i-1, i}$ and $\mathcal{A}_{n}^{i-1}$ are, respectively, given by (22) and (23). We can use (B3) successively by setting $i=1,2, \ldots, r$ to obtain the adiabatic solution of (8) with initial condition (19) as

$$
|\Psi(T)\rangle=k_{r}\left|\psi_{n}^{r}\left[\mathcal{R}\left(t_{r}\right)\right]\right\rangle=k_{r}\left|\psi_{n}^{0}\left[\mathcal{R}\left(t_{0}\right)\right]\right\rangle .
$$

Furthermore, using (B4) and (B5) recursively we find

$$
k_{r}=\mathcal{K}_{r} k_{r-1}=k_{0} \prod_{i=1}^{r} \mathcal{K}_{i}=k e^{-i \int_{0}^{T} E_{n}(t) d t} \prod_{i=1}^{r}\left\{e^{i\left(\gamma_{n}\left(\mathfrak{c}_{i-1}\right)\right)} \mathcal{G}_{n}^{i-1, i}\left(\mathcal{R}\left(t_{i}\right)\right)\right\}
$$

Note that in this construction label $i$ is cyclic in the sense that label $r$ is to be identified with 0 . For example, $\mathcal{G}_{n}^{r-1, r}=\mathcal{G}_{n}^{r-1,0}$ because $\mathcal{U}_{r}=\mathcal{U}_{0}$. The geometric phase associated with the closed curve $\mathfrak{C}$ has the form

$$
e^{i \gamma_{n}(\mathfrak{C})}=\prod_{i=1}^{r}\left\{e^{i\left(\gamma_{n}\left(\mathfrak{c}_{i-1}\right)\right)} \mathcal{G}_{n}^{i-1, i}\left(\mathcal{R}\left(t_{i}\right)\right)\right\}
$$

${ }^{1}$ A. Bohm, A. Mostafazadeh, H. Koizumi, Q. Niu, and J. Zwanziger, The Geometric Phase in Quantum Systems (Springer, New York, 2003).

${ }^{2}$ M. V. Berry, Proc. R. Soc. London, Ser. A 392, 45 (1984).

${ }^{3}$ F. Wilczek and A. Zee, Phys. Rev. Lett. 52, 2111 (1984).

${ }^{4}$ Y. Aharonov and J. Anandan, Phys. Rev. Lett. 58, 1593 (1987).

${ }^{5}$ J. Samuel and R. Bhandari, Phys. Rev. Lett. 60, 2339 (1988).

${ }^{6}$ A. Mostafazadeh, J. Phys. A 32, 8157 (1999).

${ }^{7}$ J. H. Hannay, J. Phys. A 18, 221 (1985).

${ }^{8}$ A. Mostafazadeh, J. Phys. A 31, 7829 (1998).

${ }^{9}$ J. C. Garrison and E. M. Wright, Phys. Lett. A 128, 177 (1988).

${ }^{10}$ B. Simon, Phys. Rev. Lett. 51, 2167 (1983).

${ }^{11}$ C. Dembowski, H.-D. Gräf, H. L. Harney, A. Heine, W. D. Heiss, H. Rehfeld, and A. Richter, Phys. Rev. Lett. 86, 787 (2001).

${ }^{12}$ A. A. Mailybaev, O. N. Kirillov, and A. P. Seyranian, Phys. Rev. A 72, 014104 (2005).

${ }^{13}$ W. D. Heiss and A. L. Sannino, J. Phys. A 23, 1167 (1990).

${ }^{14}$ M. Mondragón and E. Hernández, J. Phys. A 29, 2567 (1996)

${ }^{15}$ W. D. Heiss, Phys. Rev. E 61, 929 (2000).

${ }^{16}$ W. D. Heiss, Phys. Rep. 242, 443 (1994).

${ }^{17}$ A. Mostafazadeh, J. Math. Phys. 43, 6343 (2002); 44, 943 (2003) (erratum).

${ }^{18}$ A. Mostafazadeh, Ann. Phys. (N.Y.) 309, 1 (2004).

${ }^{19}$ U. Günther, F. Stefani, and G. Gerbeth, Czech. J. Phys. 54, 1075 (2004).

${ }^{20}$ W. D. Heiss, Czech. J. Phys. 54, 1091 (2004).

${ }^{21}$ W. D. Heiss, J. Phys. A 37, 2455 (2004).

${ }^{22}$ U. Günther, I. Rotter, and B. F. Samsonov, J. Phys. A 40, 8815 (2007).

${ }^{23}$ M. V. Berry, Czech. J. Phys. 54, 1039 (2004).

${ }^{24}$ T. Stehmann, W. D. Heiss, and F. G. Scholtz, J. Phys. A 37, 7813 (2004).

${ }^{25}$ I. Rotter, Phys. Rev. E 67, 026204 (2003).

${ }^{26}$ W. D. Heiss, M. Müller, and I. Rotter, Phys. Rev. E 58, 2894 (1998).

${ }^{27}$ A. I. Nesterov and F. A. de la Cruz, e-print arXiv:quant-ph/0611280.

${ }^{28}$ F. Keck, H. J. Korsch, and S. Mossmann, J. Phys. A 36, 2125 (2003).

${ }^{29}$ C. Dembowski, B. Dietz, H.-D. Gräf, H. L. Harney, A. Heine, W. D. Heiss, and A. Richter, Phys. Rev. E 69, 056216 (2004).

${ }^{30}$ G. Herzberg and H. C. Longuet-Higgins, Discuss. Faraday Soc. 35, 77 (1963).

${ }^{31}$ C. A. Mead and D. G. Truhlar, J. Chem. Phys. 70, 2284 (1979).

${ }^{32}$ C. P. Sun, Phys. Scr. 48, 393 (1993).

${ }^{33}$ T. Kato, Perturbation Theory of Linear Operators (Springer, Berlin, 1980).

${ }^{34}$ A. Mostafazadeh, Phys. Lett. A 264, 11 (1999).

${ }^{35}$ N. Steenrod, The Topology of Fiber Bundles (Princeton University Press, Princeton, NJ, 1951).

${ }^{36}$ E. H. Spanier, Algebraic Topology (Springer-Verlag, New York, 1966).

${ }^{37}$ Y. Choquet-Bruhat, C. DeWitt Morette, and M. Dillard-Bleick, Analysis, Manifolds and Physics, Part I: Basics (NorthHolland, Amsterdam, 1989).

${ }^{38}$ Ch. Miniatura, C. Sire, J. Baudon, and J. Bellissard, Europhys. Lett. 13, 199 (1990).

${ }^{39}$ G. Dattoli, R. Mignani, and A. Torre, J. Phys. A 23, 5795 (1990). 
${ }^{40}$ J. W. Brown and R. V. Churchill, Complex Variables and Applications (McGraw-Hill, New York, 1996).

${ }^{41}$ M. Nakahara, Geometry Topology and Physics (IOP, London, 1990).

${ }^{42}$ W. S. Massey, A Basic Course in Algebraic Topology (Springer, New York, 1991).

${ }^{43}$ J. M. Lee, Introduction to Topological Manifolds (Springer, New York, 2000).

${ }^{44}$ C. J. Isham, Modern Differential Geometry for Physicists (World Scientific, Singapore, 1999).

${ }^{45}$ A. Mostafazadeh, Dynamical Invariants, Adiabatic Approximation and the Geometric Phase (Nova Science, New York, 2001).

${ }^{46}$ A. Mostafazadeh, J. Math. Phys. 40, 3311 (1999). 